\title{
Reverse Total Shoulder Arthroplasty: Early Outcome and Complication Report
}

\author{
Yong-Bok Park, Sung-Weon Jung ${ }^{1}$, Ho-Young Ryu, Jin-Ho Hong, Sang-Hoon Chae, Kyoung-Bin Min², Jae-Chul Yoo \\ Department of Orthopaedic Surgery, Samsung Medical Center, Seoul, 'Department of Orthopaedic Surgery, Samsung Changwon Hospital, Changwon, \\ ${ }^{2}$ Sports Medicine Center, Samsung Medical Center, Seoul, Sungkyunkwan University School of Medicine, Korea
}

\begin{abstract}
Background: Recently, reverse total shoulder arthroplasty (RTSA) has been accepted as a main treatment option in irreparable massive rotator cuff tear with cuff arthropathy. The purpose of this study was to evaluate the early complication incidence and the preliminary clinical results of RTSAs performed in single institute.

Methods: Fifty-seven RTSAs (56 patients) were performed between April 2011 and March 2013. The indications for RTSA were cuff tear arthropathy and irreparable massive rotator cuff tear with or without pseudoparalysis. Exclusion criteria were revision, preoperative infections and fractures. At final follow-up, 45 shoulders were enrolled. Mean follow-up duration was 12.5 months (range, 6-27 months). The mean age at the time of surgery was 73.6 years (range, 58-87 years). All the patients were functionally accessed via Constant score, American Shoulder and Elbow Surgeons (ASES) score, pain and functional visual analogue scale (VAS) scores and active range of motion. Complications were documented as major and minor. Major complications include fractures, infections, dislocations, nerve palsies, aseptic loosening of humeral or glenoid components, or glenoid screw problems. Minor complications include radiographic scapular notching, hematomas, heterotopic ossification, algodystrophy, intraoperative dislocations, intraoperative cement extravasation, or radiographic lucent lines of the glenoid.

Results: The mean Constant score increased from 31.4 to 53.8 ( $p<0.001$ ). The pain and functional VAS scores improved (5.2 to $2.7, p$ $<0.001,4.0$ to $6.7, p<0.001)$ and active forward flexion improved from $96.9^{\circ}$ to $125.6^{\circ}(p=0.011)$. One or more complications occurred in $16(35.6 \%)$ of 45 shoulders, with one failure (2.2\%) resulting in the removal of implants by late infection. The single most common complication was scapular notching $(9[20 \%])$. There were $4(8.9 \%)$ axillary nerve palsies postoperatively $(n=3$ : transient $n$. palsy, $\mathrm{n}=1$ : Symptom existed at 11 months postoperatively but improving).

Conclusions: In a sort term follow-up, RTSA provided substantial gain in overall function. Most common early complications were scapular notching and postoperative neuropathy. Although overall early complication rate was as high as reported by several authors, most of the complications can be observable without compromise to patients' clinical outcome. Long term follow-up is required to clarify the clinical result and overall complication rate.
\end{abstract}

(Clin Shoulder Elb 2014;17(2):68-76)

Key Words: Shoulder; Arthroplasty; Reverse; Early outcome; Complication

\section{Introduction}

Reverse total shoulder arthroplasty (RTSA) has been developed to salvage the glenohumeral articulation damaged by infection, arthritis, or trauma. ${ }^{1,2)}$ Unfortunately, its initial design resulted in high complication and failure. ${ }^{3,4)}$ Now with marked changes in its design because of Grammont contribution in the late 1980's, the clinical outcome of RTSA has improved.,6) Reverse design moved the center of rotation (COR) distally and medially in comparison to the native glenohumeral articulation. ${ }^{7}$ The change in glenohumeral COR improves deltoid function and decreases glenoid implant-bone interface stresses and loos-

Received March 31, 2014. Revised May 26, 2014. Accepted June 3, 2014.

Correspondence to: Jae-Chul Yoo

Department of Orthopaedic Surgery, Samsung Medical Center, Sungkyunkwan University School of Medicine, 81 Irwon-ro, Gangnam-gu, Seoul

135-710, Korea

Tel: +82-2-3410-3501, Fax: +82-2-3410-0061, E-mail: neocybersky@gmail.com, shoulderyoo@gmail.com

Financial support: None. Conflict of interests: None. 
ening. Moreover, it significantly improves the function of patients with painful pseudoparalysis secondary to a massive irreparable rotator cuff tear. ${ }^{2,5)}$ Furthermore, because of recent advances in implant technology and surgical techniques, marked improvement in clinical scores, subjective patient satisfaction, complications, and failure rates has been achieved."

However, even with recent advancement, studies report wide variable clinical outcomes and complication rates reached to $75 \%{ }^{8,9)}$ Moreover, it is still considered as having much more complication rate than conventional total shoulder arthroplasty. ${ }^{3,4)}$ Furthermore, their clinical outcomes seem to have much more variance because of many factors such as expanded indications (cuff arthropathy, fracture or infection sequelae, and massive tear) ${ }^{5,6)}$ quality of remaining external rotators, bone quality, and so forth. Therefore, our early clinical results and complication rate with modern RTSAs were questionable.

The purpose of the study was to evaluate the early complication based preliminary clinical result of RTSAs performed by single surgeon in single institute. Our hypotheses were; (1) observed complication of RTSA would be similar to published rates, and (2) although the complication rate is high, most would be treated successfully without much compromise to the clinical outcome.

\section{Methods}

\section{Patients Selection}

This preliminary report is a retrospective review of $57 \mathrm{~s}^{\prime}$ consecutive RTSAs performed between April 2011 and March 2013 by one surgeon at a single institution. The indications were cuff arthropathy and massive irreparable rotator cuff tear with or without pseudoparalysis. We considered irreparable rotator cuff tear as chronic rotator cuff tears, severe atrophy of supraspinatus/infraspinatus muscle, with Goutallier grade four for supraspinatus and $3 / 4$ for infraspinatus. ${ }^{10)}$ Prior conservative treatment failed for all the patients. The exclusion criteria were insufficient follow-up period (at least six months), revision surgery, preoperative infection history, preoperative neurologic injury, and worker's compensation cases. Of the 57 shoulders, 12 cases were excluded (two follow-up loss, four insufficient follow-up period, and one incomplete axillary nerve palsy preoperatively because of shoulder dislocation, four patients with pre-operative infection history, and one patient with traffic accident just after the surgery).

Forty-five shoulders were finally enrolled in this retrospective study, which was conducted after obtaining approval from the institutional review board at our institution (IRB no. 2014-01108). The mean age at the time of surgery was $73.6 \pm 6.1$ years (range, 58-87 years). The dominant shoulder was involved in 30 patients $(66.6 \%)$, and one patients had bilateral surgery. Cemented and cementless stems were inserted in 21 and 24 shoul- ders, respectively. The mean follow-up duration was $12.5 \pm 5.6$ months (range, 6-27 months). According to Hamada-Walch classification, degrees of glenohumeral arthritis in our series were classified as stage $1-3$ in 18 shoulders and stage $4-5$ in 27 (Table 1). ${ }^{11,12)}$

Six patients had previous operations: Of these patients, 4 patients had rotator cuff repair because of rotator cuff tear. Another one patient had rotator cuff repair only because of massive rotator cuff tear and concomitant Bankart lesion with instability. Moreover, the other one patient had arthroscopic Bankart repair because of recurrent dislocation with Bankart lesion. There were no patients who had more than two operations on same side of the surgery. Fourteen patients had previous intra-articular injection more than once and 31 patients had no previous injection history before RTSA.

\section{Clinical and Radiologic Evaluation}

A clinical and radiologic assessment consisting of a structured interview, clinical examination, photographic documentation, and radiographic observation were performed preoperatively and postoperatively in each clinical visit. All the patients were functionally accessed via Constant score, ${ }^{13)}$ American Shoulder and Elbow Surgeons (ASES) score, ${ }^{14}$ Korean Shoulder Scoring System, ${ }^{15)}$ pain and functional visual analog scale (VAS) score, and active range of motion (including forward flexion, external

Table 1. Preoperative Demography

\begin{tabular}{lc}
\hline \multicolumn{1}{c}{ Variable } & Data \\
\hline Age (yr) & $73.6 \pm 6.1(58-87)$ \\
\hline Gender (male:female) & $9: 36$ \\
\hline Involved side (left:right) & $14: 31$ \\
\hline Dominant arm & $30(66.6)$ \\
\hline Stem (cemented:non-cement) & $21: 24$ \\
\hline Mean follow-up duration (mo) & $12.5 \pm 5.6(6-27)$ \\
\hline Staging of massive rotator cuff tears ${ }^{*}$ & \\
\hline Grade 1 & $2(4.4)$ \\
\hline Grade 2 & $11(24.4)$ \\
\hline Grade 3 & $5(11.1)$ \\
\hline Grade 4a & $10(22.2)$ \\
\hline Grade 4b & $7(15.6)$ \\
\hline Grade 5 & $10(22.2)$ \\
\hline
\end{tabular}

Values are presented as mean \pm standard deviation (range), number, or number (\%).

*In accordance with Hamada-Walch classification.

Grade 1: AHI $\geq 6 \mathrm{~mm}$, Grade 2: AHI $<6 \mathrm{~mm}$, Grade 3: AHI $<6 \mathrm{~mm}$ with acetabularization, Grade 4a: glenohumeral arthritis without acetabularization, Grade 4b: glenohumeral arthritis with acetabularization, Grade 5: collapse of humeral head, "cuff tear arthropathy".

AHI: acromiohumeral distance. 
rotation with the arm at the side, and internal rotation behind the back). Standardized anteroposterior, glenohumeral anteroposterior, axillary lateral, and scapular lateral and 30 degree caudal tilted view was obtained preoperatively and postoperatively.

Zumstein et al. ${ }^{8)}$ classified an intraoperative or postoperative event under problem and complication which were likely to affect the patient's final outcome or not. Problem is a type of complication, we classified problems and complications to minor and major complications, respectively. Major complications include fractures, infections, dislocations, nerve palsies, aseptic loosening of humeral or glenoid components, modular stem or polyethylene disassociations, or glenoid screw problems. Minor complications include scapular notching, hematomas, heterotopic ossifications, algodystrophy, phlebitis, intraoperative dislocations, cement extravasation, or radiographic lucent lines of the glenoid.

\section{Surgical Technique}

All procedure was performed by a single senior author (J.C.Y.). Three types of Grammont-style RTSA prosthesis were used during this period (Aequalis Reverse system type II; Tonier, Montbonnot, France) ( $n=23)$, Trabecular metal reverse shoulder system (Zimmer, Warsaw, IN, USA) $(n=7)$, Versa-dial system: standard adaptor (Biomet, Warsaw, IN, USA) $(n=15)$. If the bone stock of glenoid was not favorable for fixation due to osteoporosis or deformation of glenoid, we chose Versa-dial system which has central screw for glenoid plate fixation. And the others were used randomly according to surgeon's preference. All the patients were operated on the beach-chair position. The
$10-15 \mathrm{~cm}$ sized deltopectoral approach was used. The superior portion of the pectoralis major tendon and the coraco-acromial ligament was incised for better exposure. The subscapularis tendon was detached from the subscapularis footprint with tagging sutures. Humeral head cutting was performed with $0-20^{\circ}$ retroversion according to patient's natural retroversion and depending on the implant system. After the head cutting, the glenoid baseplate fixation was followed according to the company instrumentation manual. Glenoid base plate was placed slightly inferior to glenoid center with $10^{\circ}$ inferior tilting in all the cases. The size of the base plate was determined with available glenoid bone stock and surgeon's preference, usually the smallest diameter baseplate. In all the cases, $36 \mathrm{~mm}$ sized glenosphere was used. Using Kirschner wire, $4-5$ holes were made on anterior proximal humeral cortex for fixation of subscapularis tendon. Then, the humeral component was placed. After the insertion of trial implant, appropriate tension was determined for the case when the joint was not easily dislocated subjectively. The subscapularis tendon was repaired via transosseous sutures in most of the cases. Incised superior portion of pectoralis major muscle was repaired. A soft tissue biceps tenodesis was also performed when in the presence of the tendon. All incisions were closed over one or two suction drain.

\section{Postoperative Rehabilitation}

Postoperatively, the shoulder was immobilized with $30^{\circ}$ abduction brace for four weeks. During this period, the sling was kept for all times except for hygiene and changing clothes. Passive range of motion was initiated after removal of hemovac at

Table 2. Any Details of Complications and Treatment according to Complications

\begin{tabular}{|c|c|c|}
\hline Complication & No. & Detail and Treatment \\
\hline \multicolumn{3}{|l|}{ Major complication } \\
\hline Postoperative neuropathy & 4 & $\begin{array}{l}\mathrm{n}=3 \text { : transient, fully recovered within } 6 \text { months; } \mathrm{n}=1 \text { : symptom existed at } 11 \text { months' postoperatively, follow-up is } \\
\text { ongoing. }\end{array}$ \\
\hline Infection & 1 & Late infection at 20 months postoperatively: Implant removal and PROSTALAC insertion was performed. \\
\hline \multicolumn{3}{|l|}{ Minor complication } \\
\hline Periprosthetic fracture & 1 & Slip down, type B (The Wright and Cofield classification, Copyright Mayo Foundation) \\
\hline Acromial fracture & 1 & Spontaneous fracture: Open reduction and internal fixation using Plate and Screw performed \\
\hline Scapular notching & 9 & $\mathrm{n}=8:$ grade $1, \mathrm{n}=1:$ grade 2 \\
\hline Radiolucent line on glenoid & 2 & \\
\hline Heterotopic ossification & 2 & \\
\hline Instability-related symptom & 1 & Discomfort with "weird sound" during range of motion. There was no pain, apprehension and sign of subluxation. \\
\hline \multicolumn{3}{|l|}{ Limited range of motion } \\
\hline Ext. rotation difficulty & 6 & $\mathrm{n}=4$ : external rotation difficulties only; $\mathrm{n}=3$ : internal rotation difficulties only; $\mathrm{n}=2$ : external and internal difficulties \\
\hline Int. rotation difficulty & 5 & \\
\hline
\end{tabular}

PROSTALAC: prosthesis with antibiotic-loaded acrylic cement. 
postoperative 2 or 3 days. Active forward elevation was not permitted to protect the subscapularis repair if they had one. After four weeks, the sling was removed and tolerable active assisted passive range of motion was allowed for 4-6 weeks, and followed by active strengthening exercises.

\section{Statistical Analysis}

Comparisons of preoperative and postoperative clinical scores were performed using the paired $t$-tests or Wilcoxon signed rank test. Subgroup analysis between group A (patients with complications) and group B (patients without complications) were conducted with the Mann-Whitney U-test. All statistical analysis was performed using the IBM SPSS Statistics 20.0 software (IBM-SPSS, Armonk, NY, USA). The significance level of $p$-value was set at 0.05 .

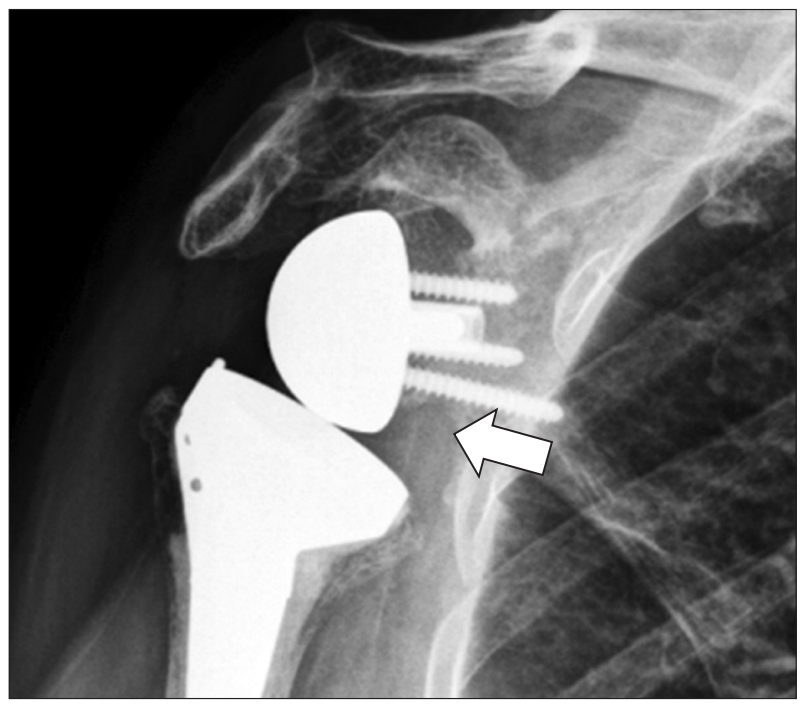

Fig. 1. Grade II scapular notching was noted at postoperative 15 months (white arrow).

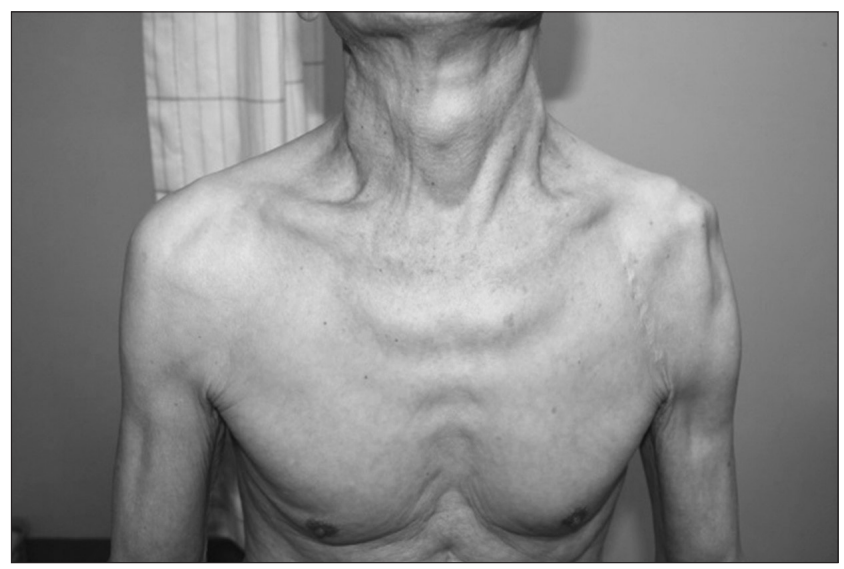

Fig. 2. The patient with axillary nerve neuropathy. Deltoid muscle wasting in involved side was noted at postoperative 11 months.

\section{Results}

\section{Complication}

One or more complications occured for 16 out of the 45 RTSAs (35.6\%) and are listed in Table 2. Minor and major complications occurred in 13 (28.9\%) and seven (15.6\%) patients, respectively, and four patients had major and minor complications simultaneously.

Scapular notching was single most common complication and was graded by the Nerot-Sirveaux grading system. ${ }^{16)}$ Nine (20\%) scapular notching were observed with eight of grade 1, and one of grade 2. However, no further complication including implant loosening was found (Fig. 1).

There were four nerve palsies existed postoperatively, and three out of which had transient axillary nerve palsies and fully recovered in sensation and function within six months. One patient still had nerve symptoms (deltoid wasting, and discomfort at forward elevation); however, he showed improvement at postoperative 11 months (forward flexion: 150 , abduction $130^{\circ}$, ASES score: 75, Constant score: 71, pain VAS: 0) (Fig. 2). No glenoid and humeral component loosening occurred; however, radiographic lucent lines of the glenoid can be seen in two cases (Fig. 3). One periprosthetic humeral fracture occurred after slip down from the front door at postoperative one month, and was treated with open reduction and internal fixation with a long locking plate (Fig. 4). One acromial fracture was developed spontaneously at postoperative six months (Fig. 5), and considered to be a fatigue fracture. Surgery was not performed, because the fracture site was peripheral and most part of the deltoid insertion was preserved. At 15 months' follow-up on this patient, active forward elevation was $130^{\circ}$, abduction was $80^{\circ}$ and pain VAS score was five. Late infection was occurred in one

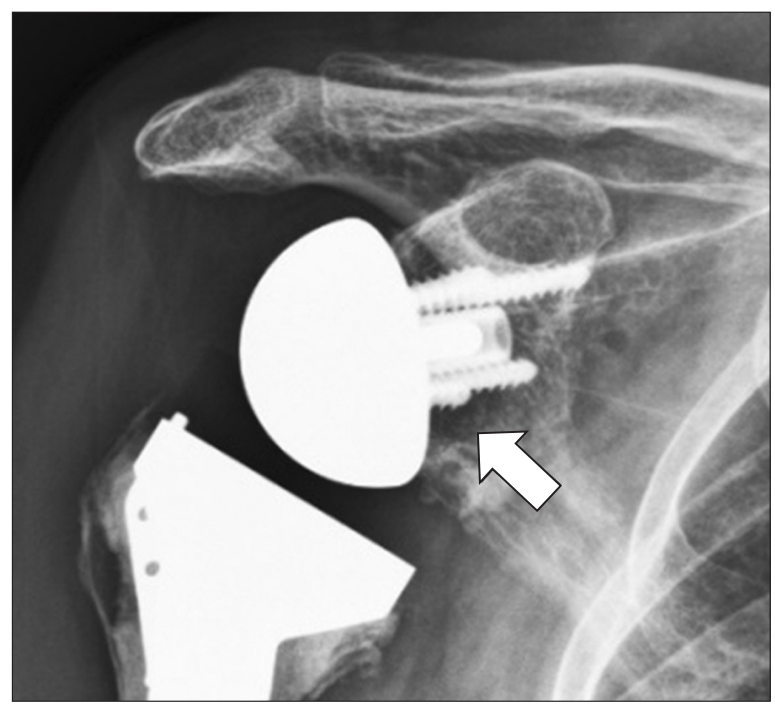

Fig. 3. Radiolucent line near glenoid component was noted at postoperative 18 months (white arrow). 


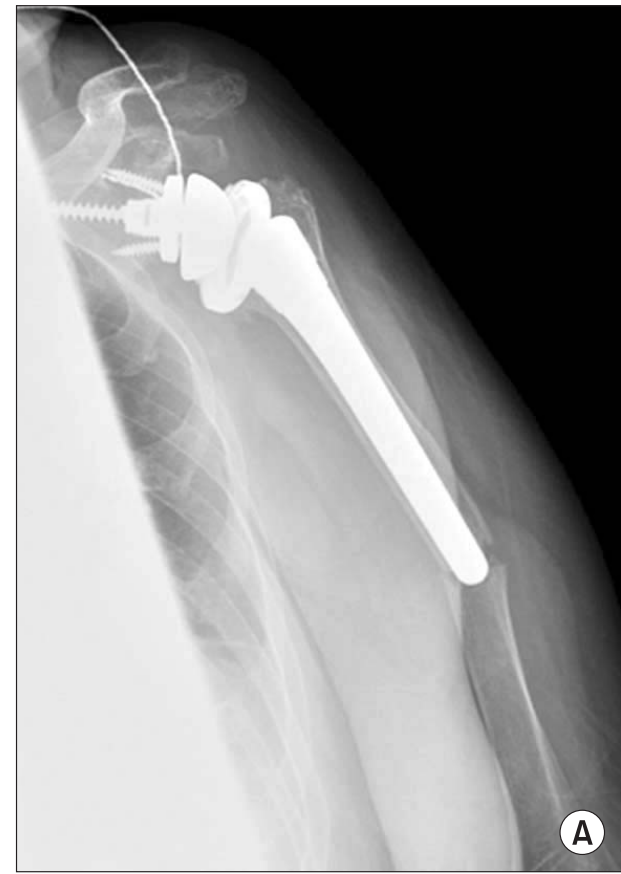

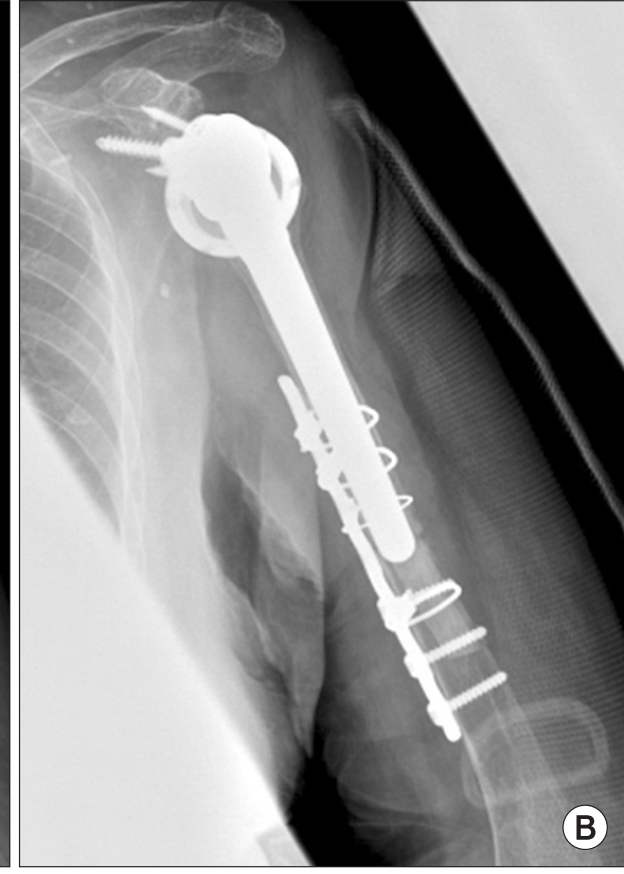

(B)
Fig. 4. (A) Periprosthetic fracture was occurred in one patient due to slip down at 1 month postoperatively. (B) The patient had open reduction and internal fixation using plate and screws.
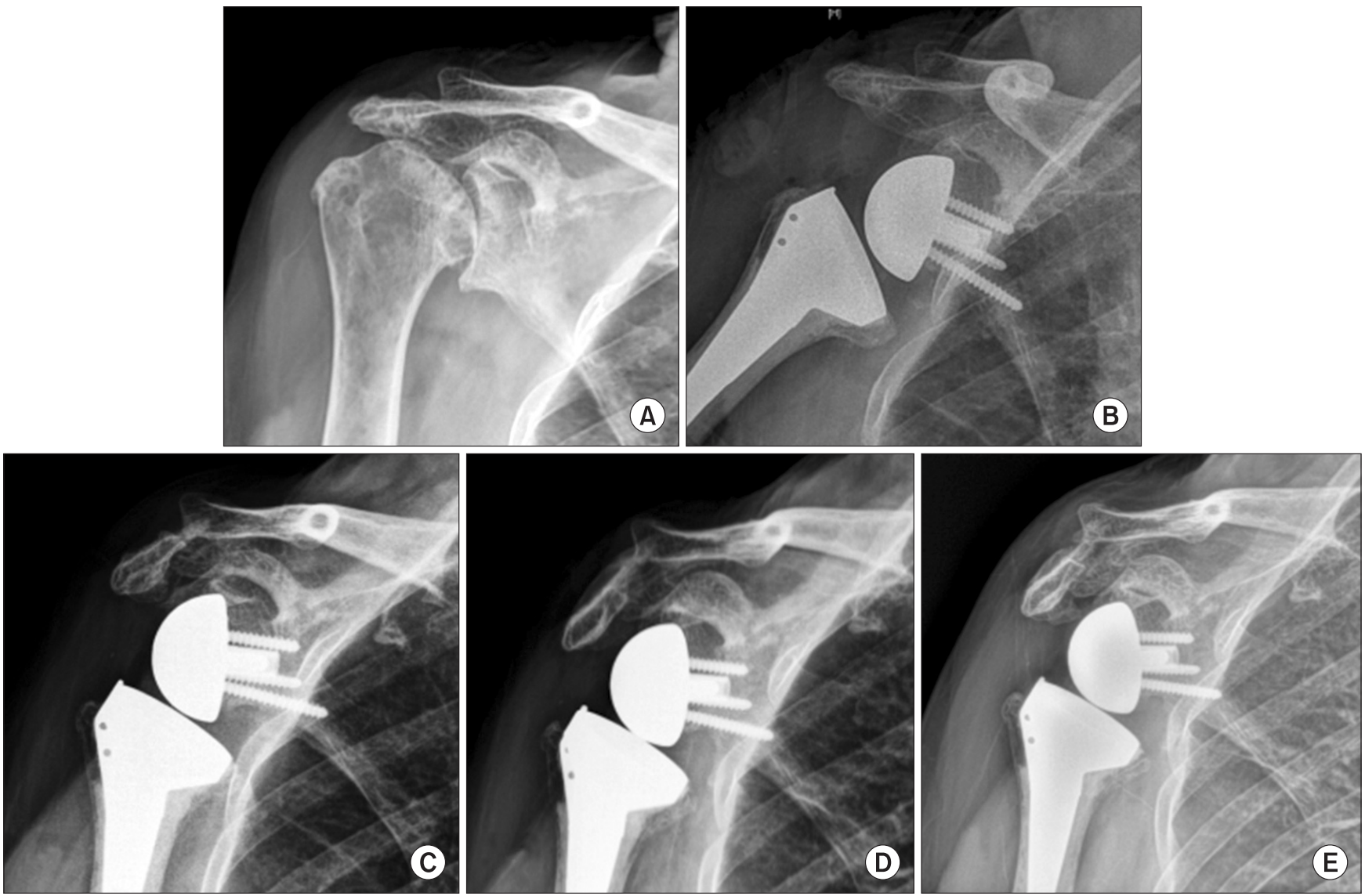

Fig. 5. Spontaneous acromial fracture was occurred in one shoulder after surgery. (A) Preoperative X-ray: Superiorly migrated humerus. (B) At 3 months postoperatively: Humerus was moved inferiorly. (C) At 6 months postoperatively: Acromial fracture was noted. (D) At 9 months postoperatively: Callus was formed around acromion. (E) At 15 months postoperatively. 

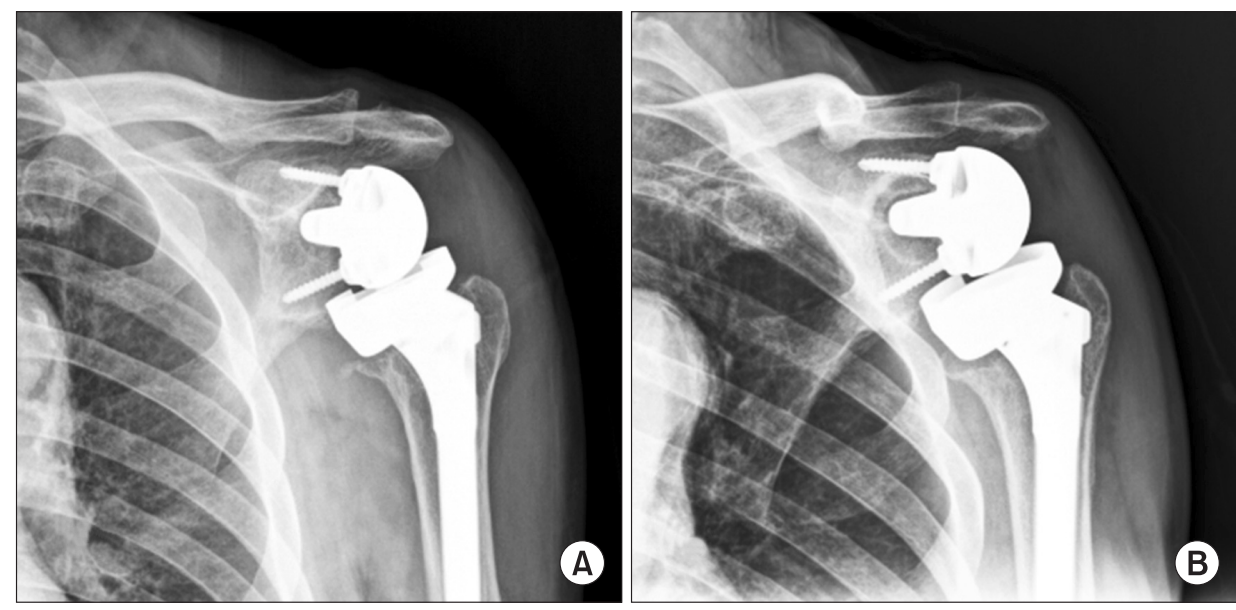

Fig. 6. The late infection was occurred in one patient. (A) Glenoid loosening was observed on 6 months' follow up x-ray. And functional score and range of motion were suddenly declined during 5 months from postoperative 6 month to 11 months. (B) Glenoid loosening was progressed. Pain, mild redness, heatness, high erythrocyte sedimentation rate and Creactive protein were observed at 20 months' follow-up.

Table 3. Preoperative and Postoperative Clinical Scores

\begin{tabular}{lccc}
\hline \multicolumn{1}{c}{ Parameter } & Preoperative scores & Postoperative scores & $p$-value \\
\hline Constant score & $31.4 \pm 15.9$ & $53.8 \pm 11.3$ & $<0.001$ \\
ASES & $33.3 \pm 16.0$ & $60.6 \pm 14.0$ & $<0.001$ \\
\hline SST & $3.0 \pm 1.9$ & $5.3 \pm 1.9$ & $<0.001$ \\
KSS & $39.6 \pm 19.7$ & $60.3 \pm 13.1$ & $<0.001$ \\
pVAS & $5.2 \pm 2.8$ & $2.7 \pm 1.6$ & $<0.001$ \\
fVAS & $4.0 \pm 2.6$ & $6.7 \pm 1.6$ & $<0.001$ \\
Range of motion $\left(^{\circ}\right)$ & & & 0.011 \\
\hline Forward elevation & $96.9 \pm 39.4$ & $125.6 \pm 16.5$ & 0.709 \\
\hline Abduction & $77.0 \pm 54.2$ & $73.3 \pm 26.0$ & 0.737 \\
\hline External rotation & $26.7 \pm 18.6$ & $27.6 \pm 12.25$ & 0.067 \\
\hline Internal rotation & $\mathrm{T} 11$ & $\mathrm{~T} 12-\mathrm{L} 1$ & \\
\hline
\end{tabular}

Values are presented as mean \pm standard deviation.

ASES: American Shoulder and Elbow Surgeons score, SST: simple shoulder test, KSS: Korean Shoulder Scoring System, pVAS: pain visual analog scale score, fVAS: functional visual analog scale score.

case. Glenoid loosening was observed on six months' follow-up X-ray. Moreover, functional score and range of motion (ROM) were suddenly declined during five months from postoperative six to 11 months. Pain, mild redness, heatness, high erythrocyte sedimentation rate (ESR) and C-reactive protein (CRP) were observed at 20 months' follow-up (Fig. 6). This patient recently received the prosthesis removal with antibiotics-mixed cement spacer insertion. Two cases of heterotopic ossification occurred on infra-glenoid neck area (Fig. 7). One patient complained discomfort with click and metallic sound during range of motion, which the patient claims as "weird sound in the case of the prosthesis moving". There was no pain, apprehension and sign of subluxation.

Hematomas, phlebitis, intraoperative cement extravasation problem, dislocation, modular stem or polyethylene disassocia-
Table 4. Clinical Score Comparison of Patients with or without Complications

\begin{tabular}{llccc}
\hline Parameter & & Group A & Group B & $p$-value \\
\hline Constant & Preoperative & $27.6 \pm 14.3$ & $28.9 \pm 17.0$ & 0.751 \\
& Postoperative & $53.0 \pm 10.5$ & $53.6 \pm 11.2$ & 0.678 \\
\hline \multirow{2}{*}{ ASES } & Preoperative & $32.5 \pm 17.8$ & $28.4 \pm 14.7$ & 0.555 \\
& Postoperative & $62.6 \pm 13.4$ & $61.6 \pm 12.9$ & 0.957 \\
\hline \multirow{2}{*}{ KSS } & Preoperative & $4.1 \pm 2.9$ & $3.3 \pm 2.3$ & 0.530 \\
& Postoperative & $5.0 \pm 1.8$ & $5.5 \pm 2.4$ & 0.512 \\
\hline \multirow{2}{*}{ pVAS } & Preoperative & $39.3 \pm 19.6$ & $39.1 \pm 19.9$ & 0.895 \\
& Postoperative & $63.4 \pm 13.4$ & $63.6 \pm 12.3$ & 0.986 \\
\hline \multirow{2}{*}{ fVAS } & Preoperative & $4.7 \pm 2.6$ & $5.2 \pm 3.0$ & 0.598 \\
\hline & Postoperative & $3.2 \pm 1.4$ & $3.1 \pm 1.7$ & 0.986 \\
\hline
\end{tabular}

Values are presented as mean \pm standard deviation.

Group A: the patients who had one or more complications, Group B: the patients who had not any complications, ASES: American Shoulder and Elbow Surgeons score, SST: simple shoulder test, KSS: Korean Shoulder Scoring System, pVAS: pain visual analog scale score, fVAS: functional visual analog scale score.

tions, and glenoid screw dislocation problems were not observed.

\section{Early Clinical Outcomes}

After excluding two patients (the patient who underwent removal of the prosthesis because of late infection, and the patient with periprosthetic fracture), overall functional scores were improved. However, the range of improvement in SST, pain VAS, and functional VAS scores were not marked. In terms of ROM, forward elevation was substantially increased $\left(96.9^{\circ}\right.$ to $125.6^{\circ}, p$ $=0.011$ ); however, abduction, external rotation and internal rotation were not significantly different. Nine patients complained 


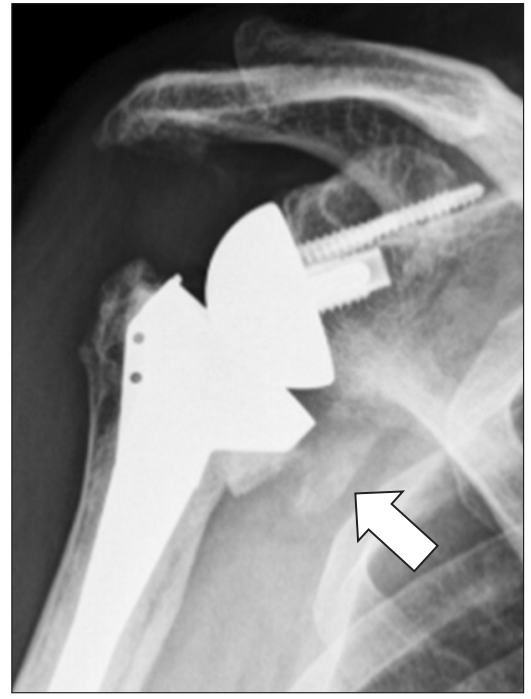

Fig. 7. Heterotopic ossification was noted at 11 months follow-up postoperatively (white arrow).

internal or external rotation difficulties (three with internal rotation difficulties only, four with external rotation difficulties only, and two with internal and external difficulties). Of total six patients who had external rotation difficulties, three patients could not at all rotate the arm externally (three with external rotation $0^{\circ}$, three with external rotation $0-20^{\circ}$ ). The preoperative and postoperative results are listed in Table 3. Table 4 lists sub-analysis of the patient with one or more complications (group A) and those with no complications (group B). No statistical differences in functional scores between groups A and B was observed.

\section{Discussion}

One or more complications occurred in 16 patients out of the 45 RTSAs (35.6\%). Our complication rate is lower than previous series, with rates reported as high as $68 \% .^{9}{ }^{9}$ Minor and major complications were $13(28.9 \%$ ) and $7(15.6 \%)$, respectively. Out of seven patients with major complication, two patients underwent surgical treatment similar to other studies where minor complications occurred more frequently and most of the major complication were treated conservatively. ${ }^{8,16,17)}$ Dislocation after RTSA was not as common as previously reported.

In our study, the incidence and severity of scapular notching $(9[20 \%])$ was lower than previously reported (range, 44-96\%). ${ }^{6,18,19)}$ However, the incidence and severity could be increased with time, the longer follow up is required to clarify the definite result. The clinical significance of scapular notching is still a matter of debate. Some studies declared that scapular notching is associated with glenoid component loosening ${ }^{16,20)}$ and has negative effect on clinical outcomes. ${ }^{16,21)}$ The largest and most comprehensive study on the subject reported no clinical effect and only one case of progressive notching leading to glenoid component loosening at 114 months postoperatively. ${ }^{22}$ Factors associated with the development of scapular notching include prosthetic design, surgical approach, positioning of the glenosphere and body mass index (BMI). ${ }^{21,23)}$ Among these parameters, lateralization of COR and inferior placement of glenosphere are associated with low rates of inferior scapular notching, improved shoulder rotation, no prosthetic instability and improved shoulder contour. ${ }^{24)}$

Internal and external rotation difficulties are other problems of Grammont-type RTSAs. In our study, the mean external and internal rotations were $27.6 \pm 12.2^{\circ}$ and $\sim \mathrm{T} 12-\mathrm{L} 1$, respectively. Nine patients complained for internal or external rotation difficulties after the operation (three with internal rotation difficulties only, four with external rotation difficulties only, and two with both difficulties). Grammont-type RTSA system (lowering the humerus and medialization of the COR at the glenoid component) has the dual advantage of tensioning the deltoid muscle to increase its functional strength, and decreasing mechanical torque at the glenoid component, thus avoiding glenoid loosening. ${ }^{2,19)}$ However, because of the medialization of COR, posterior deltoid component for external rotation and anterior component for internal rotation were decreased. Therefore, internal and external rotation difficulties occurred frequently. ${ }^{2)}$ Moreover, reduced rotational moment arms in the conjunction with the decrease in origin-to-insertion distance can cause impaired external and internal rotation. ${ }^{25)}$ Postoperative external rotation difficulty is a cause of patient's dissatisfaction. Therefore, if patient has severe external rotation lagging and insufficient teres minor muscle, surgeon should anticipate patient's dissatisfaction and explain it to the patient before surgery. If operated arm is dominant side, at least T10-L1 of internal rotation is essential for hygiene. For this reason, less degree of retroversion is favorable. Lateralization of COR improve tension of the remaining rotator cuff muscles. ${ }^{8)}$ And increased anterior and posterior recruitment can restore active internal and external rotation. ${ }^{17)}$ However, the lateralization of COR can increase shear force on glenoid component, resulting in acceleration of glenoid loosening is of theoretical concern. Some controversies exist on this issue. $\mathrm{Vi-}$ rani et al. ${ }^{26)}$ declared no difference in glenoid loosening between standard RTSA and RTSA of $10 \mathrm{~mm}$ lateralized COR. However, Boileau et al. ${ }^{24)}$ declared that bony lateralization has mechanical advantage compared to metallic lateralization, ${ }^{6,25}$ improving shoulder rotation, and no prosthetic instability. More studies are necessary to confirm this issue.

There was wide variation in the rates of postoperative hematoma between studies $(1-21 \%) .{ }^{20,27)}$ Probably because some authors only included hematomas as a complication if there was a need for a reoperation, ${ }^{5)}$ whereas others included all hematomas, even if no re-intervention was needed. ${ }^{28)}$ Our study did not have any known hematoma problems.

Neurologic complications occurred in four cases (8.9\%). All 
the cases were related to axillary nerve. Three of these were transient and one has neurologic symptom such as (Deltoid muscle wasting and difficulty in forward elevation at postoperative 11 months' follow-up). However, the symptoms were improving and more follow-up is needed. This patient's HamadaWalch stage was 4a. After superiorly migrated humerus reduced, traction injury might have occurred in this patients resulting in higher than previously reported $(0-12.2 \%)$. ${ }^{8,29}$ Neurologic complications may be attributed to intraoperative traction, manipulation of the arm, retractor placement, or relative lengthening of arm. In a systemic review of 782 cases reported by Zumstein et al. ${ }^{8)}$, nine neurologic complications were reported including axillary (two cases), radial (six cases) and musculocutaneous (one case) nerve palsies.

One (2.2\%) acromial fracture occurred spontaneously and was treated with conservative management. It was considered to be a fatigue fracture because of deltoid over-tension after the reduction of superior migrated humerus preoperatively (Fig. 5). This result is similar to the previously reported one $(0-6.9 \%)^{5,8,16)}$

Postoperative instablility, a major complication, can result in poor functional outcome and pain. Postoperative instability has been reported in a range $2.4 \%$ to $31 \%{ }^{3}{ }^{3}$ In our study, one patient complained of instability-related symptom. This patient complains weird sound and uncomfortable feeling without pain while elevating arm forward at near $90^{\circ}$ and rotating arm internally. There was no objective sign of impingement, subluxation and dislocation. We are not sure why we didn't have any distinctive dislocations. Maybe we are putting our implant too tight instead of too loose. However, we feel that it is more worrisome putting the prosthesis too tight than loose making the shoulder joint to be in the risk of acromion fracture or transient nerve palsies.

This study has several limitations. First, our follow-up is too short to make definitive estimation of the clinical outcome. Previous studies with long term follow up have shown increased complication rates with time. ${ }^{8,18)}$ Second, our small series preclude statistical analysis according to implant systems. A larger sample size will clarify the clinical result and provide greater precision for complication rate. Third, surgeon's factor is always there; therefore, our complication rate will decrease and clinical outcome will improve with accumulation. Several studies reported the learning curve for the RTSA and the complication rate tends to decrease after some numbers of operations. ${ }^{9,18)}$ Therefore, our result may show the clinical outcome and complication incidence in early phase of the first 50 surgeries of single surgeon.

\section{Conclusion}

In short term follow-up, RTSA provided substantial gain in overall function. Most common early complications were scapular notching and postoperative neuropathy. Although overall early complication rate was as high as reported by several authors, most of the complications can be observable without compromise to patients' clinical outcome. Long term follow-up is required to clarify the clinical result and overall complication rate.

\section{References}

1. Wall B, Nové-Josserand L, O'Connor DP, Edwards TB, Walch G. Reverse total shoulder arthroplasty: a review of results according to etiology. J Bone Joint Surg Am. 2007;89(7):1476-85S.

2. Boileau P, Watkinson DJ, Hatzidakis AM, Balg F. Grammont reverse prosthesis: design, rationale, and biomechanics. J Shoulder Elbow Surg. 2005;14(1 Suppl S):147S-61.

3. Cheung E, Willis M, Walker M, Clark R, Frankle MA. Complications in reverse total shoulder arthroplasty. J Am Acad Orthop Surg. 2011;19(7):439-49.

4. De Wilde LF, Audenaert EA, Berghs BM. Shoulder prostheses treating cuff tear arthropathy: a comparative biomechanical study. J Orthop Res. 2004;22(6):1222-30.

5. Boileau P, Watkinson D, Hatzidakis AM, Hovorka I. Neer Award 2005: The Grammont reverse shoulder prosthesis: results in cuff tear arthritis, fracture sequelae, and revision arthroplasty. J Shoulder Elbow Surg. 2006;15(5):527-40.

6. Gerber C, Pennington SD, Nyffeler RW. Reverse total shoulder arthroplasty. J Am Acad Orthop Surg. 2009;17(5):284-95.

7. Grammont P, Trouilloud P, Laffay J, Deries X. Concept study and realization of a new total shoulder prosthesis. Rhumatologie. 1987;39:407-18.

8. Zumstein MA, Pinedo M, Old J, Boileau P. Problems, complications, reoperations, and revisions in reverse total shoulder arthroplasty: a systematic review. J Shoulder Elbow Surg. 2011;20(1):146-57.

9. Wierks C, Skolasky RL, Ji JH, McFarland EG. Reverse total shoulder replacement: intraoperative and early postoperative complications. Clin Orthop Relat Res. 2009;467(1):225-34.

10. Goutallier D, Postel JM, Bernageau J, Lavau L, Voisin MC. Fatty muscle degeneration in cuff ruptures. Pre- and postoperative evaluation by CT scan. Clin Orthop Relat Res. 1994;(304):7883.

11. Hamada K, Fukuda H, Mikasa M, Kobayashi Y. Roentgenographic findings in massive rotator cuff tears. A long-term observation. Clin Orthop Relat Res. 1990;(254):92-6.

12. Walch G, Edwards TB, Boulahia A, Nové-Josserand L, Neyton L, Szabo I. Arthroscopic tenotomy of the long head of the biceps in the treatment of rotator cuff tears: clinical and radiographic results of 307 cases. J Shoulder Elbow Surg. 2005;14(3):238-46.

13. Constant CR, Murley AH. A clinical method of functional assessment of the shoulder. Clin Orthop Relat Res. 
1987;(214):160-4.

14. Richards RR, An KN, Bigliani LU, et al. A standardized method for the assessment of shoulder function. J Shoulder Elbow Surg. 1994;3(6):347-52.

15. Tae SK, Rhee YG, Park TS, et al. The development and validation of an appraisal method for rotator cuff disorders: the Korean Shoulder Scoring System. J Shoulder Elbow Surg. 2009;18(5):689-96.

16. Sirveaux F, Favard L, Oudet D, Huquet D, Walch G, Molé D. Grammont inverted total shoulder arthroplasty in the treatment of glenohumeral osteoarthritis with massive rupture of the cuff. Results of a multicentre study of 80 shoulders. J Bone Joint Surg Br. 2004;86(3):388-95.

17. Frankle M, Siegal S, Pupello D, Saleem A, Mighell M, Vasey M. The Reverse Shoulder Prosthesis for glenohumeral arthritis associated with severe rotator cuff deficiency. A minimum twoyear follow-up study of sixty patients. J Bone Joint Surg Am. 2005;87(8):1697-705.

18. Groh GI, Groh GM. Complications rates, reoperation rates, and the learning curve in reverse shoulder arthroplasty. J Shoulder Elbow Surg. 2014;23(3):388-94.

19. Nyffeler RW, Werner CM, Simmen BR, Gerber C. Analysis of a retrieved delta III total shoulder prosthesis. J Bone Joint Surg Br. 2004;86(8):1187-91.

20. Boulahia A, Edwards TB, Walch G, Baratta RV. Early results of a reverse design prosthesis in the treatment of arthritis of the shoulder in elderly patients with a large rotator cuff tear. Orthopedics. 2002;25(2):129-33.

21. Simovitch RW, Zumstein MA, Lohri E, Helmy N, Gerber C. Predictors of scapular notching in patients managed with the Delta III reverse total shoulder replacement. J Bone Joint Surg Am. 2007;89(3):588-600.
22. Lévigne C, Boileau P, Favard L, et al. Scapular notching in reverse shoulder arthroplasty. J Shoulder Elbow Surg. 2008;17(6):925-35.

23. Falaise V, Levigne C, Favard L; SOFEC. Scapular notching in reverse shoulder arthroplasties: the influence of glenometaphyseal angle. Orthop Traumatol Surg Res. 2011;97(6 Suppl):S131-7.

24. Boileau P, Moineau G, Roussanne Y, O'Shea K. Bony increased-offset reversed shoulder arthroplasty: minimizing scapular impingement while maximizing glenoid fixation. Clin Orthop Relat Res. 2011;469(9):2558-67.

25. Herrmann S, König C, Heller M, Perka C, Greiner S. Reverse shoulder arthroplasty leads to significant biomechanical changes in the remaining rotator cuff. J Orthop Surg Res. 2011;6:42.

26. Virani NA, Harman M, Li K, Levy J, Pupello DR, Frankle MA. In vitro and finite element analysis of glenoid bone/baseplate interaction in the reverse shoulder design. J Shoulder Elbow Surg. 2008;17(3):509-21.

27. Levy JC, Virani N, Pupello D, Frankle M. Use of the reverse shoulder prosthesis for the treatment of failed hemiarthroplasty in patients with glenohumeral arthritis and rotator cuff deficiency. J Bone Joint Surg Br. 2007;89(2):189-95.

28. Werner CM, Steinmann PA, Gilbart M, Gerber C. Treatment of painful pseudoparesis due to irreparable rotator cuff dysfunction with the Delta III reverse-ball-and-socket total shoulder prosthesis. J Bone Joint Surg Am. 2005;87(7):1476-86.

29. Bufquin T, Hersan A, Hubert L, Massin P. Reverse shoulder arthroplasty for the treatment of three- and four-part fractures of the proximal humerus in the elderly: a prospective review of 43 cases with a short-term follow-up. J Bone Joint Surg Br. 2007;89(4):516-20. 